\title{
CORRECTION OF AUTONOMIC AND COGNITIVE DISTURBANCES IN PATIENTS WITH NON-ALCOHOLIC FATTY LIVER DISEASE
}

10.36740/WLek202010117

\author{
Yelyzaveta S. Sirchak, Vasilij I. Griga, Oksana I. Petrichko \\ STATE HIGHER EDUCATIONAL INSTITUTION «UZHHOROD NATIONAL UNIVERSITY», MEDICAL FACULTY, UZHHOROD, UKRAINE
}

\begin{abstract}
The aim: To explore the features of the congnitive (CD) and neurovegetative dysfunctions (NVD) of patients with different forms of non-alcoholic fatty liver disease NAFLD (nonalcoholic fatty hepatosis (NAFH) and non-alcoholic steatohepatitis (NASH)) and type 2 diabetes mellitus (T2DM), as well as their dynamics against the background of complex therapy using armadin (2-ethyl-6-methyl-3-hydroxypyridine succinate).

Materials and methods: 50 patients with NAFLD (24 patients with NAFH and 26 patients with NASH) and T2DM were examined. Dysfunction of central and vegetative nervous system was carried out with the help of neuro-psychometric testing.

Results: The analysis of the conducted research before treatments indicates the NVD in the surveyed patients. Cognitive dysfunctions (mostly mild and moderate) was revealed in all of our patients in both groups. The most pronounced disturbances before treatments were determined in the areas of attention and concentration, memory, less pronounced - visually constructive area, especially among patients with NASH and T2DM. Stressful situations have a pronounced impact on the lives of these patients, but they are not critical enough to any issues. Patients of Group 2 before treatments had the lowest level of resistance to stress. They found a slight vulnerability even from minor impacts. Conclusions: In patients with NAFLD and T2DM, congnitive and neurovegetative dysfunctions were found, according to neuro-psychometric testing. Complex therapy using 2-ethyl-6-methyl-3-hydroxypyridine succinate is an effective and safe method for the correction of congnitive and neurovegetative dysfunctions in patients with NAFLD and T2DM.
\end{abstract}

KEY WORDS: non-alcoholic fatty liver disease, type 2 diabetes mellitus, cognitive dysfunction, vegetative dysfunction, treatment

Wiad Lek. 2020;73(10):2198-2203

\section{INTRODUCTION}

Non-alcoholic fatty liver disease (NAFLD) covers a range of diseases closely related to metabolic risk factors [1]. The common pathogenetic mechanisms for NAFLD and metabolic syndrome have been proven, and their association with type 2 diabetes mellitus, cardiovascular disease, obesity and more is evident. Overweight and insulin resistance (IR) are recognized as major risk factors for NAFLD. However, not only overeating, but also unhealthy diets, such as increased consumption of foods rich in sugar and fats, as well as lack of physical activity and genetic susceptibility are considered to be leading factors in the development of NAFLD [2].

The prevalence and severity of NAFLD is also influenced by presence of metabolic risk factors, such as overweight/ obesity and type 2 diabetes mellitus (T2DM) [1]. The prevalence of NAFLD and non-alcoholic steatohepatitis (NASH) in T2DM are $76 \%$ and $22 \%$, respectively. Furthermore, the prevalence of NAFLD correlates with the degree of impaired glucose metabolism, increasing from $27 \%$ in subjects with normal fasting glucose (fasting blood glucose $(\mathrm{FBG})<6.1 \mathrm{mmol} / \mathrm{L})$, to $43 \%$ and $62 \%$, respectively, in those with impaired glucose tolerance $(\mathrm{FBG} \geq 6.1 \mathrm{mmol}$, but $<7 \mathrm{mmol} / \mathrm{L})$ and T2DM (FBG $>7.0 \mathrm{mmol} / \mathrm{L})[3]$.

NAFLD is one of the most common chronic liver diseases worldwide [4]. NAFLD is characterised by excessive hepatic fat accumulation, associated with insulin resistance
(IR), and defined by the presence of steatosis in $>5 \%$ of hepatocytes according to histological analysis. NAFLD includes two pathologically distinct conditions with different prognoses: non-alcoholic fatty hepatosis (NAFH) and non-alcoholic steatohepatitis (NASH); the latter covers a wide range of disease severity, including fibrosis, cirrhosis and hepatocellular carcinoma. The diagnosis of NAFLD requires exclusion of both secondary causes and a daily alcohol consumption $\geq 30 \mathrm{~g}$ for men and $20 \mathrm{~g}$ for women [5].

The challenges posed by people living with multiple chronic conditions are unique for people with dementia and other cognitive impairment [6]. The term "cognitive impairment" is used to describe a deficit beyond that associated with normal aging, but not characterized as dementia. The etiology of cognitive impairment is multifactorial. Oxidative stress, immune inflammatory processes, anemia, hyperhomocysteinemia, and vitamin B12 deficiency may be involved with this decline in neurocognitive performance [7], that also can be detected in patients with non-alcoholic fatty liver disease (NAFLD) [8]. Some trialists think that non-alcoholic fatty liver disease can be well grounded regarded as cognitive-behavioral dysfunction [4].

Thus, the study of cognitive disorders, as well as the search for effective means of their correction in patients with NAFLD and type 2 diabetes is an urgent task of modern medicine. 


\section{THE AIM}

To explore the features of the congnitive and neurovegetative dysfunctions of patients with different forms of non-alcoholic fatty liver disease (non-alcoholic fatty hepatosis and non-alcoholic steatohepatitis) and type 2 diabetes mellitus (T2DM), as well as their dynamics against the background of complex therapy using armadin (2-ethyl-6-methyl-3-hydroxypyridine succinate).

\section{MATERIALS AND METHODS}

At the clinical site of the department of propaedeutic of internal diseases of the medical faculty of SHEI "UzhNU" (gastroenterology and endocrinology department of the TRCH n. a. A. Novak) during 2016-2019, 50 patients with NAFLD and T2DM were examined. The average age was $46.3 \pm 5.7$ years. They formed the main group of the examined patients (Group 1). Patients were divided into Groups, namely:

- Group 1 included 24 patients with NAFH (among them there were 14 males $(58.3 \%)$ and 10 females $(41.7 \%)$, the average age was $44.2 \pm 7.7$ years);

- Group 2 included 26 patients with NASH (among them there were 15 males (57.7\%) and 11 females ( $42.3 \%$ ), the average age was $48.1 \pm 5.3$ years).

The control group included 20 practically healthy persons (12 males $(60.0 \%)$ and 8 females $(40.0 \%)$ ), the average age was $47.6 \pm 5.8$ years.

All studies were performed with patients consent (written consent for performing appropriate diagnostic and therapeutic measures was received from all of the patients), and the methodology of their implementation were in line with the Helsinki Declaration of Human Rights of 1975 and its 1983 revised version, and in line with Europe Convention on Human Rights, as well as biomedicine and legislation of Ukraine.

All the examined patients were subject to general clinical, anthropometric, instrumental, and laboratory tests. To verify the diagnosis, attention was paid to the nature of complaints, as well as the history of the disease. In anthropometric study, height, weight, waist circumference were determined, and body mass index (BMI) was calculated (according to WHO recommendations) [9].

Ultrasound examination of the abdominal cavity was performed on all patients according to generally accepted method. Standard and biochemical blood serum tests have been performed to determine the functional state of the liver, lipid metabolism indexes and carbohydrate metabolism (glucose, insulin, glycosylated hemoglobin (HbAlc, \%) indexes.

NAFLD diagnosis was established in accordance with the Unified Clinical Protocol criteria (Order of the Ministry of Health of Ukraine dated November 6, 2014, No. 826) and EASL-EASD-EASO Clinical Recommendations for Diagnosis and Treatment of NAFLD. Herewith, the stage of the liver damage was determined on the base of surrogate laboratory liver fibrosis markers [10].

Diagnosis of type 2 diabetes mellitus has been established according to IDF recommendations (2005), as well as taking into account the criteria of unified clinical protocol (Order of Ministry of Health of Ukraine dated December 21, 2012, No. 1118) [10]. Level of severity of T2DM was evaluated according to the HbAlc (norm being up to 6.0\%).

Dysfunction of the central nervous system (CNS) in examined patients were determined using the following tests before treatment, as well as after the complex therapy:

1. The Montreal Cognitive Assessment Scale (MCAS) is used for rapid screening of cognitive impairment. It evaluates various cognitive functions: attention and concentration, executive functions, memory, language, optical-spatial activity, conceptual thinking, counting and orientation. The survey takes approximately 10 minutes. The maximum score on this scale -30 .

2. The MMSE (Mini-Mental State Examination) scale is a short mental status assessment scale used worldwide to assess cognitive function status. It is quite reliable method for primary screening for cognitive impairment, including dementia. The test result is obtained by summing the scores for each item. The maximum figure in this test -30 points, which corresponds to the highest cognitive ability. The lower the test result, the more marked cognitive deficits.

3. The technique of "Remembering $\mathbf{1 0}$ words", by A. Luria (the study of auditory memory) is used to evaluate the status of auditory memory for words, fatigue, attention activity, memorization, preservation, reproduction of arbitrary attention. With this technique determining auditory short-term and long-term memory. The number of normal memorized units is $7 \pm 2$ words.

4. Boston Stress Test, developed by scientists at Boston University Medical Center. It is necessary to answer the questions based on how often these statements are true for the subject of study. The normal reaction to stressful situations indicates the total score of 10 or less.

5. Benton's line orientation test (Benton, Hamsher, Varney, and Spreen, 1983) assesses spatial perception. The test includes 5 inclined segments. During the execution should find a line with the same slope as the control map. The Benton test is commonly used in the pathopsychological diagnosis of suspected organic brain damage, as well as clarify their degrees of severity.

6. The Schulte test is used to examine the distraction, switch, distribution, and focus of the surveyed. Allows you to determine the efficiency (exhaustion), presence or absence of attention disorders. The time of the test is taken into account (the average norm is $40-42 \mathrm{sec}$ ).

7. The Clock Drawing test is used to assess cognitive impairment and severity, as well as possible neurological and psychiatric disorders. The result is rated on a 10-point scale. The lower the score, the more pronounced is the cognitive deficit.

8. The Wisconsin Card Sorting Test (WCST) developed by Esto Berg and David Grant in 1948) - allows you to evaluate such cognitive functions as: 1) the ability to forming abstract categories; 2) the ability to switch attention when changing categories (shifting set); 3 ) ability to concentrate attention on the selected category (maintaining set); 4) the ability to use feedback (feedback utilization). The evaluation 
of results is the absolute and relative number of recognized categories, trials, errors and perseverative errors [11].

Neuro-psychometric testing carried out at intervals of 2-3 hours to restore attention and concentration ability in patients.

Determination of vegetative dysfunction (VD) was carried out with the help of O.M. Wayne's questionnaire (1998). A score greater than 15 indicated vegetative dysfunction.

The surveyed patients also determined the main indicators of hemodynamics (heart rate, blood pressure) and on the basis of the obtained data was calculated the Kerdo's vegetative index (KVI).

$\mathrm{KVI}=(1$ diastolic blood pressure $(\mathrm{DBP}) /$ heart rate $){ }^{*} 100$.

At full vegetative equilibrium (eitony) the index is in the range -10 to +10 . If sympathetic effects (sympathicotonia) are predominant, KVI will be higher than +10 , and if parasympathetic effects (vagotonia) are predominant, KVI will be below -10 [12].

For the differential diagnosis of the atherosclerotic changes in brain vessels in the examined patients, measurement of the extracranial portion of the common carotid artery posterior wall intima-media complex thickness of was performed, since this is the marker of carotid atherosclerosis. The normal thickness of the intima-media complex for all patients is less than $1.0 \mathrm{~mm}$. If the thickness index of intima-media complex of the common carotid artery posterior wall in the examined patients exceeded $1.0 \mathrm{~mm}$, the patients were excluded from the study, and the results were considered as manifestations of atherosclerotic lesions of the brain vessels.

The criteria for exclusion of patients from the study were also: type 1 diabetes, chronic hepatitis, alcoholic, viral (hepatitis B, C, D) etiologies, autoimmune hepatitis.

The scientific research was carried out within the scientific research work framework №851 "Mechanisms of the formation of complications in liver diseases and pancreas, methods of their treatment and prevention" (state registration number: 0115U001103), as well as the general department topic of the department of propaedeutics of internal diseases, as well as the scientific topic of the Department of Propaedeutics of Internal Medicine (Polymorbid pathology in diseases of the digestive system, features of pathogenesis, the possibility of correction (state registration number 0118U004365).

The provision of medical care to patients with NAFLD and type 2 diabetes was performed according to clinical protocols of treatment of the Ministry of Health of Ukraine and in accordance with local protocols. To control the glucose level in the blood serum, the administered patients were prescribed oral drugs (metformin) on the background of diet and lifestyle modification, and, if necessary, insulin therapy was performed. All patients with NAFLD and type 2 diabetes were prescribed ursodeoxycholic acid (UDHA) - Ursofalk (Dr. Falk Pharma GmbH, Germany) at a rate of $20 \mathrm{mg} / \mathrm{kg}$ body weight. UDHA treatment was continued for 6 months.

Also, all examined patients were prescribed armadin (2-ethyl-6-methyl-3-hydroxypyridine succinate) $100 \mathrm{mg}$
2 times a day for 14 days, followed by switching to tablets of $150 \mathrm{mg}$ once a day for another 14 days.

The analysis and processing of the patient examination results were carried out using the computer program "Statistics" for Windows v.10.0 (StatSoft Inc., USA) using parametric and nonparametric methods of evaluating the results obtained. By calculating statistic values, the arithmetic mean $(\mathrm{M})$ and the average error were calculated $(\mathrm{M} \pm \mathrm{m})$. The probability of differences between average values was evaluated by using the Student coefficient (normal dividation of value) or with help of Mann-Whithey test and the Pirson coefficient (for non-permanent dictates).

\section{RESULTS}

All surveyed patients had T2DM of mainly mild severity (compensated carbohydrate metabolism), which was characterized by the presence of relatively well-being, lack of hypoglycemic reactions, blood glucose on an empty stomach - up to $8.5 \mathrm{~m} / \mathrm{l}$; after a meal - up to $10 \mathrm{mmol} /$ 1, HbAlc - did not exceed $6.5 \%$.

The anthropometric study found that the prevailing majority of the examined patients of both groups had overweight or obesity of varying classification (class I-II).

The analysis of the conducted research indicates the VD in the surveyed patients of both the 1 and 2 groups according to the results of the questioning (questionnaire by O.M. Wayne), as well as the KVI. The main indicators of the functional state of the vegetative nervous system (VNS) in the examined patients are shown in table 1.

According to the obtained data, complex therapy with the use of Armadin proved to be an effective method for the correction of disorders of the autonomic nervous system in the examined patients. It should be noted more pronounced positive dynamics in VNS in patients with Group 1 (NAFH + T2DM). This was manifested by a statistically significant decrease in the vegetative state assessed on the Wayne OM scale. (up to $23.1 \pm 4.3$ points $-\mathrm{p}<0.01$ ), while in Group 2 patients this figure decreased by only $11.3 \pm 1.5$ points. A more pronounced decrease in heart rate and systemic blood pressure after treatment was also reported in Group 1.

According to the results, before treatment the vast majority of patients in both groups complained of a frequent feeling of emotional stress, mood lability, irritability, anxiety, sleep disturbance, headache, fatigue, palpitations, heart failure caused by negative emotional influences. At the same time, differences were revealed in the assessment of the degree of VD in the examined patients, namely the most pronounced VD, according to the results of the Wayne questionnaire, was diagnosed in patients with NASH in combination with type 2 diabetes (1.2 subgroup) $-54.5 \pm 4.3$ points (at $10.3 \pm 1.1$ points in the control group $-\mathrm{p}<0.01$ ), positive dynamics was found in KVI, with both groups of examined patients (Table 1).

Further analysis of the indicators of the functional status of the ANS indicates the predominance of the influence 
Table I. Dynamics of indicators of VNS functional status in examined patients against the background of complex treatment

\begin{tabular}{|c|c|c|c|c|c|}
\hline \multirow{3}{*}{ Indicator } & \multirow{3}{*}{$\begin{array}{l}\text { Control } \\
\text { group } \\
(n=20)\end{array}$} & \multicolumn{4}{|c|}{ Examined patients } \\
\hline & & \multicolumn{2}{|c|}{ Group $1(n=24)$} & \multicolumn{2}{|c|}{ Group 2 (n=26) } \\
\hline & & $\begin{array}{c}\text { before } \\
\text { treatments }\end{array}$ & $\begin{array}{c}\text { after } \\
\text { treatments }\end{array}$ & $\begin{array}{c}\text { before } \\
\text { treatments }\end{array}$ & $\begin{array}{c}\text { after } \\
\text { treatments }\end{array}$ \\
\hline $\begin{array}{l}\text { Vegetative state according to the } \\
\text { scale of Wayne OM, points }\end{array}$ & $10.3 \pm 1.1$ & $44.7 \pm 5.2^{* *}$ & $23.1 \pm 4.3++$ & $54.5 \pm 4.3^{* *}$ & $43.2 \pm 2.8$ \\
\hline Heart rate per $1 \mathrm{~min}$ & $72.7 \pm 3.2$ & $98.2 \pm 4.8$ & $81.4 \pm 3.3$ & $101.3 \pm 3.7^{*}$ & $92.4 \pm 2.1$ \\
\hline $\mathrm{SBP}, \mathrm{mm} \mathrm{Hg}$ & $110.9 \pm 8.6$ & $134.7 \pm 5.1$ & $128.8 \pm 3.2$ & $141.7 \pm 6.4^{*}$ & $132.5 \pm 2.7$ \\
\hline $\mathrm{DBP}, \mathrm{mm} \mathrm{Hg}$ & $77.7 \pm 5.2$ & $83.7 \pm 3.8$ & $80.3 \pm 2.9$ & $86.7 \pm 6.1$ & $85.3 \pm 3.4$ \\
\hline KVI & $-6.82 \pm-0.9$ & $14.6 \pm 1.1^{* *}$ & $6.5 \pm 1.3++$ & $15.9 \pm 1.4^{* *}$ & $10.3 \pm 1.2+$ \\
\hline
\end{tabular}

Note: the differences between the control group and the patients examined were significant -

${ }^{*}-p<0.05{ }^{* *}-p<0.01$; the difference between the parameters in the examined patients of Group 1 and Group 2 before and after treatment is significant: $+-p<0,05 ;++p<0,01$.

Table II. Dynamics of indicators of cognitive function in the examined patients against the background of complex treatment

\begin{tabular}{|c|c|c|c|c|c|}
\hline \multirow{3}{*}{ Indicator } & \multirow{3}{*}{$\begin{array}{l}\text { Control } \\
\text { group } \\
(n=20)\end{array}$} & \multicolumn{4}{|c|}{ Examined patients } \\
\hline & & \multicolumn{2}{|c|}{ Group $1(n=24)$} & \multicolumn{2}{|c|}{ Group $2(n=26)$} \\
\hline & & $\begin{array}{c}\text { before } \\
\text { treatments }\end{array}$ & $\begin{array}{c}\text { after } \\
\text { treatments }\end{array}$ & $\begin{array}{c}\text { before } \\
\text { treatments }\end{array}$ & $\begin{array}{c}\text { after } \\
\text { treatments }\end{array}$ \\
\hline MCAS, points & $29.7 \pm 0.6$ & $25.3 \pm 0.4^{*}$ & $28.7 \pm 0.5+$ & $23.1 \pm 0.7^{* *}$ & $25.9 \pm 0.5$ \\
\hline MMSE scale, points & $29.9 \pm 0.4$ & $24.0 \pm 0.9$ & $26.3 \pm 0.7$ & $21.3 \pm 0.5^{*}$ & $23.9 \pm 1.1$ \\
\hline $\begin{array}{l}\text { Technique of «Remembering } 10 \text { words», } \\
\text { by A. Luria, words }\end{array}$ & $9.2 \pm 1.3$ & $6.9 \pm 1.1^{*}$ & $8.1 \pm 0.4$ & $5.3 \pm 0.7^{* *}$ & $7.8 \pm 0.4+$ \\
\hline Boston Stress Test, points & $12.4 \pm 0.7$ & $47.3 \pm 0.9^{* *}$ & $23.1 \pm 0.8++$ & $56.7 \pm 3.1 * *$ & $40.5 \pm 2.9+$ \\
\hline Benton's line orientation test & $26.4 \pm 2.3$ & $23.0 \pm 1.7$ & $24.7 \pm 0.9$ & $22.1 \pm 0.9^{*}$ & $22.9 \pm 0.7$ \\
\hline Schulte's test, sec. & $41.2 \pm 3.6$ & $34.7 \pm 1.5$ & $39.3 \pm 1.0+$ & $30.7 \pm 2.2^{*}$ & $33.5 \pm 1.3$ \\
\hline The Clock Drawing test & $9.7 \pm 0.5$ & $7.7 \pm 0.4^{*}$ & $8.1 \pm 0.3$ & $6.9 \pm 0.7^{*}$ & $7.7 \pm 0.5$ \\
\hline \multicolumn{6}{|l|}{ WCST: } \\
\hline number of categories & $5.7 \pm 0.8$ & $5.2 \pm 0.4$ & $5.4 \pm 0.3$ & $5.0 \pm 0.5$ & $5.3 \pm 0.8$ \\
\hline the sum of all mistakes & $11.7 \pm 0.9$ & $16.2 \pm 0.6^{*}$ & $13.1 \pm 0.4+$ & $18.9 \pm 0.5^{*}$ & $16.6 \pm 0.9$ \\
\hline perseverative errors & $7.4 \pm 0.5$ & $6.1 \pm 0.7$ & $7.0 \pm 0.4$ & $5.0 \pm 0.6^{*}$ & $6.1 \pm 0.4+$ \\
\hline non-perseverative errors & $6.5 \pm 0.3$ & $8.3 \pm 0.4^{*}$ & $7.2 \pm 0.5+$ & $10.5 \pm 0.7^{*}$ & $9.2 \pm 0.4$ \\
\hline
\end{tabular}

Note: the differences between the control group and the patients examined were significant -

${ }^{*}-p<0.05 ;{ }^{* *}-p<0.01$; the difference between the parameters in the examined patients of Group 1 and Group 2 before and after treatment is significant: $+-p<0,05 ;++-p<0,01$.

of its sympathetic department in both groups of examined patients. It should be noted that no statistically significant difference, according to the results of KVI was observed between the survey groups.

Carrying out neuropsychometric testing before treatment made it possible to determine cognitive impairment and to establish their severity in patients with NAFLD and type 2 diabetes (Table 2).

Cognitive impairment (mostly mild and moderate) before treatment by MCAS was detected in all of our patients in both groups. The most pronounced disturbances were determined in the areas of attention and concentration, memory, less pronounced - visually constructive, especially among patients of Group 2 (NASH in combination with type 2 diabetes). Analyzing the results obtained on the MMSE scale before treatment, we can see that patients with NAFLD and T2DM have impaired intelligence in the form of mild dementia, with a maximal deviation from the norm in patients with and NASH in combination with type 2 diabetes $(21.3 \pm 0.5$ points $) \mathrm{p}<0,05$. Also the attenuation of attention, increased fatigue, forgetfulness is indicated by the results of A. Luria's test in all examined patients with a maximum deviation from the norm before treatment in Group 2.

Reduced levels of stress persist according to the results of the Boston test in patients of Group $1(\mathrm{p}<0.01)$. Stressful situations have a pronounced impact on the lives of these patients, but they are not critical enough to any issues. Patients with NASH combined with T2DM have the lowest level of resistance to stress $(56.7 \pm 3.1$ points $-\mathrm{p}<0.01)$. 
They found a slight vulnerability even from minor impacts.

Expressed memory impairment and inability to focus on specific tasks for a long time, reduced performance, rapid fatigue were diagnosed using the Benton, Schulte and Clock Drawing tests. At the same time, only in patients of Group 2 the difference of the received data was statistically significantly different from such indicators in the control group ( $p<0.05)$ according to the results of Benton and Schulte tests.

The inability to switch attention effectively when the environmental conditions are changing was revealed by the WCST in the form of an increase of perseverative responses while performing this test, which may adversely affect verbal functions in the examined patients with NASH and type 2 diabetes.

So patients with NAFLD and type 2 diabetes have VNS dysfunction, as well as cognitive impairment with maximal abnormalities in patients with NASH before treatments.

Course therapy with 2-ethyl-6-methyl-3-hydroxypyridine succinate has been shown to be effective in correcting cognitive impairment in patients with NAFLD and T2DM. At the same time, a more pronounced positive dynamics was established by the results of repeated neuropsychometric testing in patients of Group 1 . There was a statistically significant increase in MCAS scores (up to $28.7 \pm 0.5$ points $-\mathrm{p}<0.05)$, which was manifested mainly with improved concentration and attention. Re-examination according to the results of the Benton's line orientation test and Schulte's test in the examined patients also indicate the improvement of memory and concentration ability.

After the treatment, an improvement in the response to stressful situations was registered in both groups of the studied patients according to the Benton's line orientation test. Along with this, it should be noted more pronounced positive dynamics in patients with NAFH and T2DM (by $24.2 \pm 0.8$ points $-\mathrm{p}<0.01)$.

Receiving a comprehensive treatment with Armadin contributed to the improvement of verbal functions in the examined patients of both groups according to the results of WCST re-evaluation.

\section{DISCUSSION}

The results of our studies show discernible CD in patients with incorporate pathology, namely NAFLD and T2DM, that progress proportional to duration of their disease. The usage of neuropsychometric tests for detecting cognitive, psycho- and neurovegetative dysfunctions in patients with NAFLD and T2DM due to results of our searching is an effective method of diagnostic the dysfunction of nervous system.

It should be noted the effectiveness of 2-ethyl-6-methyl-3-hydroxypyridine succinate in the complex treatment of patients with NAFLD and T2DM. 2-ethyl-6-methyl-3-hydroxypyridine succinate succinate is a heteroaromatic antioxidant that has a wide range of pharmacological effects: increases the body's resistance to stress, has an anxiolytic effect that is not accompanied by drowsiness and muscle relaxant effect; has nootropic properties, prevents and reduces memory disorders that occur due to aging and under the influence of various pathogenic factors; exhibits antioxidant and antihypoxic properties; increases concentration and efficiency. The drug improves the metabolism of brain tissues and their blood supply, improves microcirculation and rheological properties of blood, reduces platelet aggregation. Stabilizes the membrane structures of blood cells, as well as reduces the content of total cholesterol and low-density lipoprotein.

The wide therapeutic spectrum of action is due to its antioxidant and membrane-protective activity. It inhibits lipid peroxidation, increases superoxide oxidase activity, increases the lipid-protein ratio, reduces membrane viscosity; modulates the activity of membrane-bound enzymes (calcium-independent phosphodiesterase, adenylate cyclase, acetylcholinesterase), receptor complexes (benzodiazepine, GABA, acetylcholine), which enhances their ability to bind to ligands, and promotes the preservation of transmission. Armadine increases the level of dopamine in the brain.

Thus, as shown by the results of our studies due to the above mechanisms of action on the body, complex therapy with Armadin improves autonomic and cognitive impairment in patients with NAFLD and T2DM, which is manifested by increased attention, concentration and attention in these patients. It should be noted that a more pronounced positive trend was obtained in the group of patients with the initial stages of liver damage, namely in patients with NAFH and T2DM

\section{CONCLUSIONS}

1. In patients with NAFLD and T2DM, congnitive and neurovegetative dysfunctions were found, according to neuro-psychometric testing.

2. Complex therapy using 2-ethyl-6-methyl-3-hydroxypyridine succinate is an effective and safe method for the correction of congnitive and neurovegetative dysfunctions in patients with NAFLD and T2DM

\section{REFERENCES}

1. Stepanov Yu.M., Nedzvetskaya N.V., Yagmur V.B. et al. Non-alcoholic fatty liver disease: features of metabolic changes at different stages of the disease. Gastroenterology. 2018; 52 (1): 13-18. (in Ukrainian)

2. Brandt A., Hernández-Arriaga A., Kehm R. etal. Metformin attenuates the onset of non-alcoholic fatty liver disease and affects intestinal microbiota and barrier in small intestine. Scientific Reports. 2019; 9: 6668.

3. Gan L., Chitturi S., Farrell G.C. Mechanisms and Implications of AgeRelated Changes in the Liver: Nonalcoholic Fatty Liver Disease in the Elderly. Current Gerontology and Geriatrics Research. 2011: Article ID 831536, 12 pages

4. Macavei B., Baban A., Dumutrascu D.L. Psychological factors associated with NAFLD/NASH: a systematic review. European Review for Medical and Pharmacological Sciences. 2016; 20: 5081-5097.

5. EASL-EASD-EASO Clinical Practice Guidelines for the Management of Non-Alcoholic Fatty Liver Disease. Obesity Facts. The European Journal of Obesity. 2016; 9: 65-90. 
6. Snowden M.B., Steinman L.E., Bryant L.L. et al. Dementia and cooccurring chronic conditions: a systematic literature review to identify what is known and where are the gaps in the evidence. Int. J. Geriatr Psychiatry. 2017; 32 (4): 357-371.

7. Gesualdo G.D., Duarte J.G., Zazzetta M.S. et al. Cognitive impairment of patients with chronic renal disease on hemodialysis and its relationship with sociodemographic and clinical characteristics. Dement Neuropsychol. 2017; 11 (3): 221-226.

8. Morettia R., Carusoa P., Gazzin S. Non-alcoholic fatty liver disease and neurological defects. Annals of Hepatology. 2019; 18: 563-570.

9. WHO: Global Database on Body Mass Index. Available from: http://apps. who.int/bmi/index.jsp?introPage=intro_3.html

10. European Association for the Study of the Liver (EASL), European Association for the Study of Diabetes (EASD) and European Association for the Study of Obesity (EASO) EASL-EASD-EASO Clinical Practice Guidelines for the management of non-alcoholic fatty liver disease. Journal of Hepatology. 2016; 64: 1388-1402.

11. Ahutina T.V., Melikjan Z.A. Neuropsychological testing: a review of current trends. On the occasion of the 110th birthday of A. R. Luria. Clinical and special psychology/2012; 2: 1-20. www.psyjournals.ru/ psyclin

12. Nasonova T.I., Klimenko O.V., Kolosova T.V. et al. Neurovegettive and cognitive impairments associated with anxiety in middle-aged patients with underlying cerebrovascular diseases. Simejna Medicina. 2017; 2 (70): 97-100. (in Ukrainian).
The scientific research was carried out within the scientific research work framework №851 "Mechanisms of the formation of complications in liver diseases and pancreas, methods of their treatment and prevention" (state registration number: 0115U001103), as well as the general department topic of the Department of Propaedeutics of Internal Diseases, as well as the scientific topic of the Department of Propaedeutics of Internal Medicine (Polymorbid pathology in diseases of the digestive system, features of pathogenesis, the possibility of correction (state registration number 0118U004365).

\section{ORCID and contributionship:}

Yelyzaveta S. Sirchak: 0000-0001-6738-0843 ${ }^{A, C, F}$

Vasilij I. Griga: 0000-0003-3666-8910

Oksana I. Petrichko: 0000-0001-5420-6424 ${ }^{B, E}$

\section{Conflict of interest:}

The Authors declare no conflict of interest

\section{CORRESPONDING AUTHOR Yelyzaveta S. Sirchak \\ Department of Propedeutics of Internal Diseases, SHEl «Uzhhorod national university» Sqr. Narodna, Ukraine, 88000, Uzhhorod, Ukraine tel: +380509761794 \\ e-mail: sirchakliza777@gmail.com}

Received: 30.06 .2020

Accepted: 28.08 .2020

A - Work concept and design, B - Data collection and analysis, C - Responsibility for statistical analysis,

D-Writing the article, $\mathbf{E}$ - Critical review, $\mathbf{F}$ - Final approval of the article 